\title{
ARTIGO
}

\section{TECNOLOGIAS DIGITAIS E ANÁLISE DO REGIME DE INFORMAÇÃO PARA A PROMOÇÃO DA SAÚDE COLETIVA}

\author{
DIGITAL TECHNOLOGIES AND ANALYSIS OF THE INFORMATION REGIME FOR THE \\ PROMOTION OF COLLECTIVE HEALTH
}

${ }^{1}$ Doutora em Comunicação pela Universidade Federal do Rio de Janeiro (UFRJ).

E-mail: marianelidagomez@gmail.com

\section{ACESSO ABERTO}

Copyright: Esta obra está licenciada com uma Licença Creative Commons Atribuição 4.0 Internacional. $(\mathrm{cc}) \mathbf{B Y}$

Conflito de interesses: A autora declara que não há conflito de interesses.

Financiamento: Este trabalho tem vinculações com a pesquisa "Governança das ações de informação", desenvolvida com o apoio do Conselho Nacional de Desenvolvimento Científico e Tecnológico (CNPq).

Declaração de Disponibilidade dos dados: Todos os dados relevantes estão disponíveis neste artigo.

Recebido em: 20/09/2018.

Revisado em: 01/10/2018.

Aceito em: 10/10/2018.

\section{Como citar este artigo:}

GONZÁLEZ DE GÓMEZ, M. N. Tecnologias digitais e análise do regime de informação para a promoção da saúde coletiva. Informação em Pauta, Fortaleza, v. 3, número especial, p. 9-29, nov. 2018. DOI: https://doi.org/10.32810/25253468.ip.v3iEspecial.2018.39711.9-29.
Maria Nélida González de Gómez ${ }^{1}$

\section{RESUMO}

São complexos e múltiplos os recursos e fluxos informacionais que cotidianamente visam a vincular o atendimento à saúde, com as expectativas e necessidades da população brasileira. As tecnologias digitais são competentes para processar e transladar todas as linguagens, mas mediadas por códigos, normas e padrões. Elas tanto inscrevem suas lógicas operacionais sobre os contextos em que atuam, como são contaminadas pelas demarcações regulatórias organizacionais. 0 desenho e implementação das tecnologias digitais estará condicionado pela pluralidade de tempos e agências locais que devem ser articulados, além de todos os desafios culturais, econômicos e políticos, próprios de um macro sistema de saúde pública. Nesse quadro, são propostas teorias sobre regimes de informação, considerando que sua flexibilidade, transversalidade e pluralismo epistemológico, ofereceriam maior liberdade analítica e descritiva. do ponto de vista da reconstrução de ações, sistemas e, recursos de informação e de paradigmas de políticas tecnológicas. Como cuidado e fortalecimento das premissas democráticas, cabe lembrar que o sucesso da implementação das tecnologias de informação e suas possibilidades inovadoras, requer mais que o acesso e a transparência das informações, um processo continuo de aprendizagem crítico sobre modelos, fontes e dispositivos de informação em e para a saúde, sua produção, efeitos e validade. Esse processo de aprendizagem deve incluir a todos, os desenvolvedores de política e os gestores, os pesquisadores e os profissionais da clínica, mas 
muito especialmente o cidadão-usuário e protagonista do SUS.

Palavras-chave: Tecnologias digitais. Regime de informação. Saúde coletiva.

\begin{abstract}
There are complex and multiple resources and information flows that daily aim to link health care with the expectations and needs of the Brazilian population. Digital technologies are competent to process and translate all languages, but are mediated by codes, norms and standards. They inscribe their operational logics on the contexts in which they act, but are contaminated by organizational regulatories demarcations. The design and implementation of digital technologies will be conditioned by the diversity of local times and agencies that must be articulated, as well as all the cultural, economic and political challenges inherent in a
\end{abstract}

macro public health system. In this framework, theories on information regimes are proposed, considering that their flexibility, transversality and epistemological pluralism, would offer greater analytical and descriptive freedom, from the point of view of the reconstruction of actions, system and information resources and paradigms of technological policies. As care and empowerment of democratic assumptions, it is worth remembering that the success of the implementation of information technologies and their innovative possibilities requires more than access and transparency of information, a continuous process of critical learning about information models, sources and devices in and for health, its production, effects and validity. This learning process should include all, policy makers and managers, researchers and clinic profissionals, but especially the citizen-user and protagonist of SUS.

Keywords: Digital technologies. Information system. Collective health.

\section{INTRODUÇÃO}

Recuperamos um texto de Canguilhem que ilustra os complexos caminhos entre os estados existenciais de saúde, felicidade, bem-estar ou doença, e a exegese médica, que vão da "saúde sem ideia" (mas não sem significados) do cotidiano das vivências, à sua translação em plurais ideias do corpo e da saúde construída pela clínica, os exames laboratoriais e imagéticos, os laboratórios de pesquisa científica, a epidemiologia, a gestão hospitalar, as políticas públicas, o atendimento e os cuidados, nossos bancos de dados indexados e os tesauros das linguagens informacionais.

A reflexão hermenêutica de Ayres (2007) ecoa um texto de Canguilhem que escolhemos reproduzir como ponto de partida destas reflexões:

A verdade de meu corpo, sua própria constituição ou sua autenticidade de existência, não é uma ideia suscetível de representação. [...] Essa saúde sem ideia, ao mesmo tempo presente e opaca é, no entanto, o que suporta e valida, de fato e em última instância, para mim mesmo e também para o médico enquanto meu médico, o que a ideia do corpo, isto é, o saber médico, pode sugerir como artifício para sustentá-la. Meu médico é aquele que aceita, de um modo geral, que eu o instrua sobre aquilo que só eu estou fundamentado para lhe dizer. (...) meu médico é aquele que aceita que eu veja nele um exegeta, antes de vê-lo como reparador (CANGUILHEM, 2005 apud AYRES, 2016, p. 174, grifo nosso). 
Nas sociedades contemporâneas são longos os caminhos que vão das vivências de nossos corpos e sua expressão em nossas linguagens biográficas e memórias culturais, até as interpretações da exegese clínica, as representações generalizadoras do conhecimento científico e as mediações e procedimentos padronizados da engenharia biomédica, de todos os quais esperamos um retorno nos cuidados e no atendimento, como metas da gestão e das políticas públicas da saúde. É esse longo caminho, com plurais vielas, territórios emuralhados e passagens em construção, que as tecnologias digitais se propõem mediar e otimizar, oferecendo suas potências vinculantes, tanto às formas da medicina personalizada e em contextos privados, como aos macros sistemas públicos da saúde coletiva.

Nesse cenário, as tecnologias digitais tiveram uma expansão e uma energia de reformulação dos contextos informacionais, perpassando todas as esferas da vida humana, pelo qual Sandra Braman (2004), entre outros, as caracteriza por sua capacidade de produzir convergências.

A transversalidade econômica, social e cultural das ações de informação e comunicação, teria sido prevista por Zurkowski, que usa o termo "infostructure", em 1984, e vai ser projetada sobre as esferas públicas, na década de 1990, no discurso antecipatório de Al Gore, vice-presidente dos Estados Unidos, que usa a expressão "General Information Infraestructure”, para referir-se à implantação das redes digitais, na reunião da International Telecommunication Union (ITU), em Buenos Aires.

Para Sandra Braman, as tecnologias digitais modificariam de modo radical os modos do fazer (making) do homem contemporâneo. ${ }^{i i}$ Como meta-tecnologias, se caracterizam por aumentar o grau de liberdade com que os homens podem atuar nos mundos social e material e por seus efeitos de convergência. Elas aconteceriam não só pela reunião de tecnologias de computação e de comunicação, mas por gerar inúmeras convergências entre as tecnologias e outros materiais e processos sociais. A autora enumera, assim, a convergência do simbólico com o material, por meio da escrita; das tecnologias simbólicas com a energia, no século XIX; entre as tecnologias de computação e comunicação graças à digitalização, a meados do século XX; das tecnologias digitais e o mundo orgânico, com a biotecnologia, no século XXI.

Ao mesmo tempo, as tecnologias e meta-tecnologias estão associadas a utilização consensual de padrões e protocolos, e implicam programas coordenados de ação 
coletiva, em seu desenvolvimento e uso: elas requerem e possibilitariam, ao mesmo tempo, o desenvolvimento de modos específicos de coordenação e interação.

É nessa direção, de convergência, vinculação e coordenação, que tem sido esperada sua intervenção nas diferentes esferas da atividade social, da produção de conhecimento científico ao atendimento e cuidados da saúde.

Tomemos como exemplo um dos dispositivos possibilitados pelas tecnologias digitais, interativas, hipermídia, dotadas de plurais recursos analíticos, as plataformas de pesquisas translacionais, que, conforme Silva, Morel e Moraes (2012), teriam dois momentos principais: (1) "transferência de novos conhecimentos adquiridos no laboratório sobre os mecanismos da doença para o desenvolvimento de novos métodos de diagnóstico, terapia e prevenção, bem como seus primeiros testes em humanos," e (2) "tradução dos resultados de estudos clínicos na prática clínica diária e na tomada de decisão em saúde" (SILVA et al., 2012, p. 349). Canuel et al. (2014), citam como exemplos as BRISK, ou cBio Cancer Portal, entre outras, que se propõem reunir, interoperacionalizar e facilitar a análise de volumosas redes de dados que visam a integrar os dados sobre a contextura molecular da doença com os dados clínicos dos pacientes.

Nos últimos anos, surgiram novas tendências no gerenciamento e análise de dados clínicos e ômicos. Várias opções foram tomadas para produzir soluções relativas a "métodos de informática que conectam entidades moleculares a entidades clínicas" (ALTMAN, 2012 apud CANUEL et al., 2014, p. 281, tradução nossa, grifo nosso).

As consultas de telessaúde e o histórico clínico dos pacientes, digitalizado, seriam assim peças chaves na produção de convergência entre diferentes ações e recursos do atendimento à saúde. Entre as múltiplas alternativas, os aplicativos de smartphones facilitam a formação de grupos de pacientes, que interagem e trocam experiências sobre sintomas e tratamentos, assim como se mantêm em contato com os agentes de saúde e o atendimento médico.

Com o objetivo de dar visibilidade a algumas das condições que facilitam ou inibem a ancoragem das tecnologias digitais na concepção e desempenho do SUS, propomos utilizar de modo descritivo-analítico o conceito de regimes de informação, no escopo e abrangência dos estudos sociais e políticos da informação e da comunicação. 


\section{CONCEITUANDO "REGIME(S) DE INFORMAÇÃO": DA ABORDAGEM DE REGIME GLOBAL E EMERGENTE AOS “REGIMES DE POLÍTICAS”}

Num primeiro momento, cabe considerar algumas abordagens dos regimes de informação, como recurso de leitura transversal das dinâmicas que envolvem conhecimento, comunicação e informação, em contextos heterogêneos e plurais, como possível contribuição à imersão das concepções e implementação das tecnologias digitais em macro sistemas de saúde, nos espaços sociais, culturais, políticos e econômicos de sua implementação.

\footnotetext{
"Regime de informação" seria o modo de produção informacional dominante em uma formação social, o qual define quem são os sujeitos, as organizações, as regras e as autoridades informacionais e quais os meios e recursos preferenciais de informação, os padrões de excelência e os modelos de sua organização, interação e distribuição, vigentes em certo tempo, lugar e circunstância, conforme certas possibilidades culturais e certas relações de poder. (GONZÁLEZ de GÓMEZ, 2003, p. 61).
}

Em tempos em que a economia parecia falar mais alto que a política, nos estudos da informação, Bernd Frohmann (1995) introduz o termo "regime de informação", com a definição inicial a seguir:

Chamaremos de "Regime de Informação" o conjunto mais ou menos estável de redes formais e informais nas quais as informações são geradas, organizadas e transferidas de diferentes produtores, através de muitos e diversos meios, canais e organizações, à diferentes destinatários ou receptores de informação, sejam estes usuários específicos ou públicos amplos (FROHMANN, 1995).

Os usos heurísticos permitidos pelo caráter transversal do conceito ofereciam perspectivas inovadoras aos estudos das dinâmicas info-comunicacionais, dando visibilidade às relações plurais e diversas de sua ocorrência, em contextos intermediáticos, trans-institucionais, interorganizacionais e intersociais.

Para Sandra Braman (2004), a inspiração e ancoragem de seus conceitos estaria nas teorias políticas desenvolvidas nos estudos das relações internacionais. 0 uso do termo regime, nas relações internacionais, buscava entender e analisar as cadeias decisórias na esfera das relações internacionais, que excediam os contornos geopolítico dos Estados Nacionais, mas carecendo de sustentação institucional; o recurso aos discursos, normas e direitos, oferecia princípios menos formais nos quais ancorar e justificar à nova ordem das relações mundiais. A apropriação do conceito, por analogia, ofereceria um caminho para o entendimento de um Emergent Global Information Policy 
Regime (2004), conforme três necessárias mudanças de perspectiva: de estatuto; da escala e da agência, e da unidade de análise.

Primeiro, a mudança de estatuto político das questões de informação: após ter sido consideradas como políticas de baixo impacto, adquirem uma nova categoria de "políticas de alto nível", associadas as políticas internacionais.

Em segundo lugar, a mudança de escala, tinha colocado em crise a identificação da agência das relações internacionais, seja o Estado Nacional ou as sociedades, ou um complexo estados/sociedade. Nessa conjuntura, Braman (2004) encontra, na abordagem construtivista dos regimes internacionais de políticas, a vantagem de deixarse em aberto a categoria de agência do regime de políticas, de modo que sua qualidade e identificação serão reconhecidos a posteriore, nas esferas de elaboração e implementação das políticas.

As mudanças de escala e de agência, são acompanhadas pela demanda de mudança da unidade de análise. Braman $(2004,2006)$, nos remete à vigência um pluralismo sociológico, numa perspectiva meta-teórica que acolhe mais de uma abordagem e ponto de vista: as teorias das redes, entendendo as relações antes como interpessoais e setoriais, que estruturais, faz da interdependência uma das principais variáveis das análises de redes; o aporte teórico das abordagens das plurais governamentalidades, de Foucault, aplicado ao estudo dos Estados, entre outros (BRAMAN, 2004).

Nos deteremos em duas questões em que a teoria do regime introduz nova ênfase ou perspectiva. Primeiro, a mudança de estatuto político das questões de informação. Consideradas questões estratégicas, enquanto associadas às políticas científicas e tecnológicas e aos projetos de segurança e desenvolvimento, sob regimes centrados no Estado, ou politicamente invisibilizadas como "meios" ou "recursos" da economia, as questões de informação passariam a estar associadas, de maneira generalizadora e abstrativa, a uma "sociedade da informação". As tecnologias digitais permitiriam esse uso ampliado e genérico do termo "informação", por sua própria capacidade de convergência e pelo envelopamento de técnicas e tecnologias "pré digitais", pelo qual podem ser denominadas como "meta-tecnologias".

No ponto de partida, com essa perspectiva, entenderíamos que não se trata de analisar como as tecnologias digitais se aplicam ou divulgam na área de saúde, mas sim 
entender como se manifestam e estão acontecendo, nos múltiplos cenários, práticas e circunstâncias da saúde pública.

A segunda condição da emergência do conceito seria a mudança da unidade de análise das questões de informação, sendo essa a característica que será mais explorada neste trabalho. Sandra Braman caracteriza essa unidade de análise como regime global emergente de políticas de informação, reafirmando suas potencialidades de convergência, de perpassar o material e o simbólico, e de estar em constante mutação.

\begin{abstract}
Assim como, nas duas últimas décadas, diversas vertentes da economia se reuniram na economia da informação (Lamberton, 1998), questões políticas tão distintas quanto os fluxos globais de informação, comunicação e cultura, atualmente também estão se unindo, num único regime global emergente de política de informação. Esse regime é "global" porque envolve atores estatais e não-estatais, e "emergente" - um conceito retirado da teoria dos sistemas complexos adaptativos - porque tanto o tema do regime quanto suas características estão ainda em evolução. (BRAMAN, 2004, p. 12, tradução nossa).
\end{abstract}

Com essa orientação, Braman recorre às teorias de política internacional, escolhendo a Stephen Krasner (1982), reconhecido pesquisador das relações internacionais, como principal interlocutor. Krasner (1982) considera o regime político como uma das matrizes paradigmáticas dos estudos de política internacional. Para o autor

Os regimes internacionais são definidos como princípios, normas, regras e procedimentos de tomada de decisões de determinada área das relações internacionais em torno dos quais convergem as expectativas dos atores. (KRASNER, 1982, p. 83).

Sandra Braman (2004) destaca a relevância outorgada por Krasner aos quadros normativos, como dimensão constitutiva de um regime político. Para Braman, no cenário atual, estaríamos justamente frente a um regime global emergente de informação, sendo que sua própria constituição é resultado dos vácuos normativos e jurídicos que se estabelecem nas arenas internacionais, quando se trata de arbitrar conflitos, resolver situações problemáticas, tomar decisões e estabelecer acordos, sem dispor de um tribunal ou de um "guarda-chuva" normativo compartilhado e validado pelas partes envolvidas, fora das ordens legais dos diferentes Estados envolvidos. Essas zonas anárquicas da ordem global poderiam ser percebidas como um lócus preferencial em que interagem, e se reformulam mutuamente, os regimes de informação e os regimes de políticas. 
No mundo contemporâneo, são correntes os problemas que desbordam mais de uma arena de políticas, e cuja reconstrução requer considerar mais de uma moldura normativa, são muitas outras as questões que demandam a superar ação de fronteiras, além das relações internacionais. Com expectativas tanto críticas como descritivas, outras abordagens das ciências políticas se questionam sobre quais as unidades de análise deveriam ser utilizadas no entendimento de problemas que, em diferentes escalas geográficas, sociais e políticas, perpassam mais de uma jurisdição ou fronteira epistêmica, institucional, jurisdicional, corporativa.

Neste estudo, recorremos a uma outra perspectiva sobre as unidades de análise, contrapondo o que seus autores denominam "regimes de políticas" (policies), as formações formação de subsistemas de políticas-estruturas de governança que precisariam ser explicadas, antes que ser categorias explicativas.

"Subsistemas de políticas" teriam como referência uma composição de atores e recursos formada em torno de um mesmo marco institucional e normativo, demandando visões convergentes de autoridade e de valor. Cabe assim perguntar por seu funcionamento e seus efeitos, quando um subsistema tem que interagir com outros atores e subsistemas, em contextos complexos de problemas e programas de ação.

Munidos dessas perspectivas sobre a unidade de análise, que permitiria agregar planos interpretativos à concepção dos regimes de informação, enquanto configurações transversais, espera-se reconstruir algumas das condições e possibilidades das tecnologias digitais, no escopo e abrangência do Sistema único de Saúde e da saúde coletiva. Nessa nova direção, recorremos aos estudos de Joachim e May $(2010,2013)$ e de Kent Eaton (2017), entre outros.

\section{DOS REGIMES DE POLÍTICAS ÀS QUESTÕES DE GOVERNANÇA}

Joachim e May (2010, p. 303) apresentam o conceito de "regimes de política" (policy), como uma abordagem aplicável a diferentes e situações problemas, incluindo os "regimes de cruzamento de fronteiras" (boundary spanning regimes), que seriam arranjos de governo que perpassam múltiplos subsistemas.

Para Joachim e May (2010), os subsistemas, também denominados por alguns monopólios políticos, domínios políticos ou sub-governos, se caracterizam por : “a) uma 
coalizão estabilizada de interesses"; b) "uma ideia" ou "imagem dominante", que outorga consistência as abordagens dos problemas entre as partes interessadas; c) uma configuração institucional dominante, que estrutura os arranjos de poder e o fluxo de informações. (JOACHIM; MAY, 2010, p. 4). Tomar os subsistemas como unidade de análise teria efeitos negativos, tanto analíticos como pragmáticos, já que or grandes problemas contemporâneos frequentemente cruzam mais de um domínio, subsistema de política ou programa de ação, de modo que alguns falam dos "problemas perversos" das arenas sociais. Das drogas ao meio ambiente, a pobreza, a poluição, a saúde, alguns dos problemas que tem mobilizado os atores políticos aconteceram além de, e não no interior de, um único subsistema político.

Cada um dos subsistemas relevantes fornece uma lente separada através da qual visualiza os problemas. Cada um deles também tem maneiras diferentes de lidar com problemas, pois têm históricos de elaboração de políticas separados e servem a interesses diferentes. Devido a essas diferenças, alcançar a unificação desejada entre os elementos de diversos subsistemas de políticas para qualquer questão de alargamento de fronteiras é o calcanhar de Aquiles do governar [governamento]. Nossa discussão sobre os regimes de política está fundamentalmente relacionada aos arranjos de governança que promovem ações unificadas entre os subsistemas. (JOACHIM; MAY, 2010, p. 7, tradução nossa, grifo nosso).

Para os autores, uma questão central, nestes casos, é justamente superar ora a inercia, ora a perspectiva segmentadora, ora os efeitos excludentes do predomínio da expressão de interesses de um único subsistema de políticas. Analisar uma situação problemática heterogênea, na perspectiva de um subsistema, inibe a percepção de condições e possibilidades que se constituem justamente nos empecilhos e dinâmicas de cruzamento e superação das fronteiras subsistemas. Seria, justamente, essa junção de possibilidades e condições, constituídas além das delimitações singulares de cada subsistema (paradigmáticas, normativas, corporativas), um dos objetos privilegiados das análises políticas, assim como uma questão central para os fazedores de políticas, visando promover a governança e a elaboração de programas coordenados de ação.

Se os subsistemas políticos se constroem dentro dos limites de uma demarcação institucional e em geral relativamente estabilizada, os regimes de políticas se constroem justamente perpassando aquelas mesmas fronteiras institucionais (JOCHIM; MAY, 2010, p. 6).

Esse transbordamento dos subsistemas e o desvelamento das relações transfronteiras é algo que se tende a resgatar pelo conceito de regime de informação. 
Logo, a leitura transversal dos regimes de informação, no cruzamento de fronteira e nos interstícios das configurações de mais de um subsistema ou configurações mono institucionais de recursos e sistemas de informação, parece conveniente para a análise de fluxos info-documentários do escopo e abrangência em e além dos subsistemas de políticas.

Em decorrência dessa perspectiva, a abordagem dos regimes de informação ofereceria instrumentos heurísticos e analíticos para uma reformulação transinstitucional do ponto de vista e demarcação metodológica dos objetos das práticas e pesquisa de informação. Essa flexibilidade interpretativa poderia colocar-se em jogo para estabelecer condições e possibilidades das tecnologias digitais e seus potenciais de transversalidade e convergência, no escopo e abrangência do Sistema único de SaúdeSUS.

Para os autores (JOACHIM; MAY, 2010; MAY; JOACHIM, 2012), regimes de políticas designa um construto que não tem visibilidade referencial (como subsistema ou arena de políticas), mas que se identifica pelos componentes que o constituem, a saber, "as ideias, arranjos institucionais e alinhamentos de interesses" (MAY; JOACHIM, 2012).

0 valor do construto é descritivo e analítico. Como um construto descritivo, a noção de um regime de políticas é útil para fornecer um mapa conceitual dos arranjos de governo para tratar de um determinado problema ou conjunto de problemas. ... As contribuições analíticas das perspectivas de regime revelam como as políticas públicas estabelecem processos de retroalimentação que reformulam o ambiente político e, por sua vez, afetam a eficácia das políticas públicas. (MAY; JOCHIM, 2012, p. 4).

May e Joachim (2012) destacam, além da leitura transversal e sincrônica das relações entre subsistemas, uma linha de acompanhamento vertical (ou diacrônico) de reformas políticas que interagem e se reforçam através do tempo, dando como exemplo a construção das leis americanas que lidam com a poluição, elaboradas entre 1969 e 1976 (MAY; JOACHIM, 2012, p. 4).

Os conceitos de limites ou fronteiras, reintroduzidos de diferentes pontos de vista nos estudos contemporâneos das ciências humanas e sociais, requerem uma análise cuidadosa, e são um desafio para ações e sistemas de informação, porque se fronteiras sinalizam a dissociação de duas partes, indicam também que as mesmas partes estão "irreversivelmente dissociadas", e são de alguma maneira muito relevantes umas para as outras.

O conceito de regime de políticas é ressignificado por Eaton (2017), em 
análises de política comparada, analisando situações e possibilidade de justaposição de regimes. Para Edward Gibson, aconteceria a justaposição de regimes políticos em "situações em que dois níveis de governo com jurisdição sobre o mesmo território operam sob regimes políticos diferentes", que, para Eaton, seriam um regime político nacional e um regime político nacional subalterno (GIBSON, 2005, p. 103 apud EATON, 2017, p. 39). Diferenciando, de acordo com May e Jochim, um regime político de "regimes de políticas", Eaton considera a constituição, na América Latina, de regimes de políticas discordantes, numa mesma unidade geopolítica, com orientações simultaneamente estatistas e neoliberais, ou privatistas e orientadas ao mercado (EATON, 2017, p. 40).

Sem entrar no debate de Eaton sobre a aplicação dos modelos de justaposição de dois regimes políticos (dissonância entre níveis nacional e subnacional de governo), destacamos sua abordagem sobre a justaposição de regimes de políticas, que poderia ter uma aplicação mais flexível e quiçá setorial:

\begin{abstract}
Mais recentemente, a mudança para o estatismo em nível nacional e o repúdio aos modelos neoliberais que ocorreram no turno de esquerda desde 2000 desencadearam a defesa de projetos mais favoráveis ao mercado em nível municipal ou departamental, como na Argentina, Bolívia e Equador. Assim, em contraste com a justaposição do regime político, em que os democratas são sempre nacionais e os autoritários sempre subnacionais, a justaposição do regime de políticas (policies) é caracterizada por uma dinâmica mais flexível na qual liberais e estatistas podem ocupar posições nacionais ou subnacionais. (EATON, 2017, p. 1940).
\end{abstract}

May, cientista político norte-americano, junto a diferentes co-autores, tem como objeto de preocupação o desenho de um cenário de segmentação do Estado em subsistemas de políticas, em configurações em redes e policentricas. O ponto de vista é analisar arranjos governamentais de elaboração e implementação de políticas públicas, em "regimes de cruzamento das fronteiras" (boundary spanning regimes). Entendendo que, por sua construção, um regime de informação está constituído por micropolíticas, políticas indiretas ou invisibilizadas, oferecendo um mapa genealógico da formação de políticas públicas, seria possível homologar as dinâmicas dos regimes de informação com as modalidades dos regimes de políticas. Podemos assim aferir novos planos de complexidade dos regimes de informação, entendidos como unidades de análise que visam à reconstrução dos cruzamentos de fronteiras entre diferentes subsistemas e contextos institucionais de ações e recursos de informação, gerando tanto tensões como 
possibilidades não obvias de resolução de problemas.

Isto permitiria uma releitura das dinâmicas e fluxos da informação, cujas unidades relacionais e complexas perpassariam as fronteiras geradas por matrizes epistêmicas multi, inter e transdisciplinares, pela estruturação diferenciada da gestão das unidades de atendimento e da clínica, dos laboratórios de pesquisa biomédica e epidemiológica, das agências do Estado, como Secretarias e Ministérios de saúde. Do mesmo modo, seria impensável que ações em saúde não implicassem fluxos informacionais que perpassaram questões urbanas, ambientais, educacionais, financeiras e legislativas.

Sob essa perspectiva, ganhariam visibilidade as tensões geradas tanto pela relação de fronteira entre subsistemas, como pela justaposição de tendências ou regimes num domínio comum em que atuam e operam diferentes atores, linguagens profissionais, marcos institucionais, interesses e paradigmas da saúde e da gestão e política em saúde. Tal seria, por extensão, o caso de atrito e justaposição de modelos, códigos, padrões, quando eles remetem ora aos marcos referenciais do Estado, ora aos agentes e regras dos mercados, gerando tensões no desenho e uso de equipamentos e tecnologias digitais na esfera de saúde.

Já Jugen Habermas chamava a atenção sobre a formação dos Estados modernos e contemporâneos, e suas tendências a configurações administrativas segmentadas em plurais subsistemas, operando, cada um deles, em torno de um tema problema. Com relativa autonomia, cada subsistemas geraria códigos e padrões próprios que se convertem em fronteiras, dos subsistemas entre sim e dos subsistemas com as sociedades a que pertencem. Cada um constrói sua ideia da sociedade, e desenvolve suas molduras discursivo-normativas, sem ponderar os efeitos e custos de cada subsistema sobre os outros (HABERMAS, 1996).

Trata-se de tensões que não são exclusivas nem do Brasil nem da América Latina, mas se manifestam e requerem cuidados específicos em cada contexto de análise. Neste caso, trata-se de sinalizar alguns dos condicionantes dos regimes vigentes de informação, que afetam e propiciam a incorporação das tecnologias digitais na esfera da saúde, visando a promover o cumprimento dos objetivos do Sistema Único de Saúde. 


\section{OS REGIMES DE INFORMAÇÃO, OS MACRO-SISTEMAS DE SAÚDE E AS TECNOLOGIAS DIGITAIS NO SUS}

Recorremos, neste ponto, ao trabalho de dois especialistas da área de informática, da Universidade de UIO, Noruega, com trabalhos de pesquisa acerca de grandes sistemas de informação em saúde, Bendik Bygstad e Ole Hansethiii. Os pesquisadores noruegueses têm realizado estudos sobre grandes sistemas de saúde, especialmente na Europa, com ênfase nas infraestruturas dos sistemas de informação em saúde. Tratandose de países com uma história significativa em investimentos e implementação de sistemas públicos de saúde, como Inglaterra e Noruega (BYGSTAD; HANSETH, 2016), as questões e problemas apresentados poderiam ser de alguma serventia nas considerações sobre o papel das tecnologias digitais na consolidação e aprimoramento do SUS.

Entre as questões principais - ou como a mais abrangente -, Bygstad e Hanseth destacam a existência de uma assimetria entre as expectativas afirmativas sobre o desempenho das tecnologias de informação (TI) nos macros sistemas de saúde, e a segmentação dos serviços que implementam essas tecnologias, em diferentes países.

Atualmente, existe uma lacuna entre as altas expectativas em relação à e-saúde e o status fragmentado dos serviços de TI. Em resposta a essa situação, as autoridades de saúde de muitos países tem demandado uma estratégia de governança central de TI e arquitetura empresarial, mas muitos grandes programas experimentam tensões. Nossa pergunta de pesquisa é: como podemos entender e administrar as tensões de programas de larga escala em ehealth? (BYGSTAD; HANSETH, 2016, p. 1).

Bystad e Hanseth afirmam que a maioria dos países optam pela combinação de duas abordagens, para ordenar a informatização dos grandes sistemas de saúde: soluções pela arquitetura de TI; soluções pela governança de TI.

Enquanto o alinhamento pela governança remete ao planejamento, outorgamento de prioridades e coordenação de múltiplas atividades nos domínios das ações e das práticas das agências envolvidas, uma arquitetura remete à concepção e implementação integrada de padrões e processos na esfera de sua intervenção. Bygstad e Hanseth problematizam a executabilidade e oportunidade de uma visão holística radical, e em geral dos modelos "top-down", aplicada aos mega sistemas de e-saúde ${ }^{\mathrm{iv}}$, propondo a busca de soluções mais flexíveis e com soluções diferenciadas, ora locais, ora 
centralizadas.

Edwards et al. (2007), citado pelos autores, consideram que existem três principais tipos de tensão nas ciber-infraestruturas, afetando-as em suas características orientadas à equidade e sustentabilidade: tempo, escala, agência (EDWARDS et al., 2007 apud BYGSTAD; HANSETH, 2016). Para Bygstad e Hanseth (2016), essas tensões se manifestam de forma diferenciada quando olhadas do ponto de vista da governança ou da arquitetura das tecnologias de informação (Quadro 1).

\begin{tabular}{|c|l|c|l|}
\hline \multicolumn{4}{|c|}{ Quadro 1 - Tensões em infraestruturas de informação } \\
\hline & Tempo & Escala & Agência \\
\hline IT & $\begin{array}{c}\text { Entre a utilidade a } \\
\text { curto tempo e a } \\
\text { evolução em } \\
\text { tempos longos }\end{array}$ & $\begin{array}{c}\text { Entre controles } \\
\text { descentralizados } \\
\text { ou centralizados }\end{array}$ & $\begin{array}{c}\text { Entre ações } \\
\text { planejadas e } \\
\text { emergentes }\end{array}$ \\
\hline $\begin{array}{c}\text { Entre padrões } \\
\text { IT }\end{array}$ & $\begin{array}{c}\text { Entre escala } \\
\text { elobal e } \\
\text { estáveis, de longa } \\
\text { duração, e }\end{array}$ & $\begin{array}{c}\text { Entre formas } \\
\text { fracas ou forte } \\
\text { dintecture }\end{array}$ \\
& dinâmicas flexíveis & locais & acoplamento \\
\hline
\end{tabular}

Fonte: BYGSTAD; HANSETH, 2016, p. 4, tradução nossa.

Para Bygstad e Hanseth (2016), da revisão da literatura e de suas próprias pesquisas, poderiam inferir-se algumas orientações acerca da implementação da governança, e a concepção da arquitetura de infraestruturas de informação, em grandes sistemas de e-saúde, tendo como princípio de ponderação a estabilidade relativa dos setores e processos em análise e avaliação. Para os autores, ainda que não se tenham suficientes e generalizáveis evidências empíricas, entre os elementos mais estáveis estariam "informações básicas do paciente e alguns outros registros básicos, como medicamentos e prescrições"; de estabilidade media, rotinas bem estabelecidas, como as dos laboratórios, e menos estáveis seriam equipamentos utilizados no atendimento dos pacientes, sujeitos a constante inovação (BYGSTAD; HANSETH, 2016, p .16).

A experiência internacional com tecnologias digitais em grandes sistemas de saúde traz à luz uma situação de tensões que, conforme a concepção de infraestrutura, não podem ser ignoradas nem se pode pretender eliminar por inteiro, já que dessas tensões decorrem também processos de inovação e o desenvolvimento de novas e melhores soluções.

Modelos holísticos e centralizadores, visando a uma integração rigorosa, 
poderiam não ser tão bem-sucedidos como modelos flexíveis, com estabelecimento de soluções adequadas as plurais situações e necessidades. Na literatura consultada, surgem indicações sobre a importância de dispor de diferentes soluções de governança, diferenciando-se os processos e situações estáveis, que permitem padronização e planejamento de longa duração, das situações e processos em constante mutação ou imprevisíveis, para os quais não se possui parâmetros estáveis de comparação ou definição, e devem requerer soluções descentralizadas e locais, conforme recursos e necessidades.

Nesse sentido, as abordagens que trabalham com o conceito de regimes de informação, por sua flexibilidade e certo pluralismo epistemológico, parecem oferecer uma liberdade analítica e descritiva de maior eficácia, do ponto de vista da reconstrução de ações, sistemas, recursos e tecnologias de informação, que abordagens que trabalham com modelos únicos, como "campos" ou redes interpessoais.

Em síntese, informação e as tecnologias digitais que compõem as diferentes expressões da e-saúde não deveriam ser consideradas em sua neutralidade funcional, fora dos contextos culturais, políticos, econômicos e sociais de sua concepção e implementação, ainda que as tensões resultantes da digitalização da infraestrutura informacional da saúde sejam de extensão internacional: a) em parte, alguns problemas surgem porque os recursos disponíveis reproduzem estruturas da informação concebidas em configurações prévias, organizacionais ou tecnológicas, tendo que ser adequadas a novas ofertas e demandas; b) a concepção e implementação de dispositivos e redes de e-saúde seriam muitas vezes definidos como subsistemas e serviços que se especializam num certo tipo de eventos e demandas de atendimento, o que tende a diminuir os efeitos distributivos sobre outras áreas de necessidades e demanda; c) outras vezes, trata-se de unidades de inovação delimitadas por jurisdições estatais (por exemplo, estaduais ou municipais), que ainda que sigam rotinas bem sucedidas e tenham efeitos benéficos, não "conversam" com outras unidades, e suas realizações são menos aproveitadas na melhoria do macro sistema da saúde; d) aconteceria frequente instabilidade dos acordos, padrões e contratos, sendo que a negociação de condições e demandas é compartilhada por atores públicos e privados, com diferentes regimes de regulação e de definição de valor. 


\section{O REGIME DE INFORMAÇÃO EM SAÚDE E SEUS AGENTES: o protagonismo do SUS}

Um regime de informação, como outros regimes de políticas, só ganha visibilidade através de seus componentes. Num regime de informação, são componentes ou dimensões do regime, seus agentes, seus contextos normativos, sua infraestrutura e disponibilização de meios e artefatos, assim como certa definição da autoridade epistêmica e de padrões de reconhecimento e validação da informação. Entre esses componentes, um lugar privilegiado é ocupado pelos agentes do regime, que são os que possuem autoridade decisória, epistêmica e normativa. Os agentes dos regimes de informação seriam aqueles que intervém e dinamizam ações, práticas e processos de informação.

Num regime de informação do SUS são plurais e diversos os agentes que, em múltiplas formações intersubjetivas, trazem experiências e competências de diferente ordem, biomédica, sanitarista, de informática e informação em saúde. Empreendedores e categorias profissionais especializados, tem um papel importante na construção permanente de um sistema público de saúde coletiva, como profissionais da saúde e profissionais de informação, técnicos, gestores, provedores de equipamentos e serviços, pesquisadores. Em cada caso, exercem suas capacidades e funções conforme protocolos, formulários, planilhas ocupacionais, vocabulários oficiais, a serem conferidos nas disposições legais e trabalhistas e plausíveis de comparação em estatísticas internacionais, e requerem credenciamento diferenciado.

Do paciente à clínica, dos serviços de atendimento aos laboratórios, os macros sistemas de saúde funcionam conforme gramáticas complexas, por vezes por acoplamentos frouxos entre as partes, por vezes conforme um entreamado de funções estabilizadas por tradições e formatos institucionais, numa superposição de tempos e padrões que favorecem sua invisibilidade.

Os regimes de informação, que são parte constitutiva e condicionante desses macros sistemas de atendimento à saúde, eles mesmos estão sujeitos (ou não), a segmentações, a herança indiscernível das práticas do passado, as prioridades ocasionais que acontecem a posterior como desvios. Ninguém ignora as dificuldades, a demandar o esforço e comprometimento de todos os participantes do Sistema, para o estabelecimento de arquiteturas e modelos de governança atentos aos princípios e finalidades do SUS, com comunicabilidade e transparência. E não seria suficiente, sem 
indagar, com igual prioridade, pela inclusão informacional e comunicacional dos agentes, que sendo categorizados como pacientes, "sujeitos de uma saúde sem ideia", são os patronos e destinatários de um Sistema público de saúde.

Em princípio, "um regime de informação se caracteriza por sua complexidade e por sua não transparência imediata, e seriam as políticas intencionais as que orientam ações e decisões destinadas "a preservar e a reproduzir, ou a mudar e substituir um Regime de Informação", podendo ser tanto políticas tácitas ou explícitas quanto micro ou macro políticas (GONZÁLEZ DE GÓMEZ, 1999).

Num espaço interdisciplinar de profissionais de saúde e de informação, aparece em toda sua magnitude o problema da infraestrutura infocomunicacional de um macro sistema de saúde pública, assim como a pluralidade de esforços e realizações nessa direção, das plataformas translacionais, a telessaúde, aos grupos de pacientes no WhatsApp.

A dupla entrada, de cidadão-usuário do SUS, não poderia deixar de ser afetada, por vezes pela insuficiência, por vezes pela complexidade e pluralidade dos meios e recursos de informação, que geram efeitos adicionais de intransparência e podem gerar efeitos excedentes de exclusão.

De que maneira a pluralidade de fontes informacionais, concebidas em diferentes momentos, campanhas, sub-agências, comunidades epistêmicas, se distribuem, justapõem e vinculam (ou não), nos dispositivos digitais ou em espaços infocomunicacionais acessíveis?

O protagonismo do SUS, sua aposta na universalização do atendimento à saúde, requerendo a participação intencional e comprometida de seus agentes, deve ter como um de seus aliados a autonomia informacional da população que atende: além das informações em e para a saúde, existe um espaço trans-informacional e comunicacional, que requer ações diferenciadas de formação e aprendizagem informacional e comunicacional sobre os próprios regimes e políticas de informação e comunicação em saúde, incluindo os recursos e ofertas das novas tecnologias. (RENEDO et al., 2017).

Cabe perguntar como podemos avançar além da concepção de subsistemas estruturados em torno de um núcleo identitário de institucionalização: disciplinar, corporativo, geopolítico, normativo; e assumir compromissos e tomar decisões ponderando mais de um contexto de justificação: ético, político, econômico, científico, 
cultural. Como podemos trabalhar para desbordar os cercos da informação envelopada por gramáticas proprietárias ou administrativas, gerando transversalidade que possam transformar a alteridade de práticas e modelos em oportunidades de aprendizado.

Cabe perguntar quais os modelos de negócios para equacionar a necessidade de lidar com tecnologias proprietárias ao mesmo tempo que atender aos requisitos de estabilidade, duração, padronização, acesso, próprios de um sistema público e gratuito de saúde.

Quando as tecnologias ganham capacidades de agregação, convergência, transversalidade, cabe perguntar como elas potencializam as ações de informação em saúde, que as constituam como recursos sociais - visando aos cuidados e atendimento da saúde dos coletivos, otimizando conhecimentos, competências, recursos socio-técnicos, e promovendo formas colaborativas de organização e trabalho.

Podemos, porém, ir mais longe, e perguntar-nos sobre adequada apropriação, pelos pacientes-cidadãos, dos saberes sobre o arranjo e distribuição das informações em saúde, os mapas metainformacionais e comunicacionais que ofereçam não só acesso às informações em saúde, sobre serviços de atendimento e sobre cuidados, mas que também possam implementar uma aprendizagem - gradual mas generalizada e permanente - sobre os atuais regimes de informação em saúde, dentro e fora do SUS considerando atores como a ANS - Agencia Nacional de Saúde Suplementar, ou as políticas de alimentação escolar. A educação é uma ferramenta fundamental na consolidação das conquistas democráticas.

\section{CONCLUSÃO}

A voracidade inovadora das tecnologias digitais nos deve levar a valorizar as plurais manifestações dos saberes e das memórias. As tecnologias, nelas mesmas, tanto dependem como contribuem com a construção de espaços não maquínicos de configuração de algo em comum, de aprendizagem e de construção de novas linguagens, informacionais, educacionais, políticas: thesauros desprivatizados enriquecidos pela imaginação linguística de plurais atores coletivos. 


\section{AGRADECIMENTOS}

Agradecemos a Drํㅗ. Isa Maria Freire e Drํㅗ. Marcia Teixeira Cavalcanti pela leitura e comentários sobre o texto.

\section{REFERÊNCIAS}

\section{AANESTAD, M. et al. Information} infrastructures within European health care: Working with the installed base. Switzerland: Springer, 2017.

AKKERMAN, S. F.; BAKKER, A. Boundary Crossing and Boundary Objects. Review of educational research, [S.l.], v. 81, n. 2, p. 132-169, 2011. Disponível em: <http://rer.aera.net>. Acesso em: 10 jul. 2018.

AYRES, J. R. C, M. Georges Canguilhem e a construção do campo da saúde coletiva brasileira. Intelligere-Revista de História Intelectual, São Paulo, v. 2, n. 1, p. 139-155, 2016.

BRAMAN, S. Defining information: an approach for policy-makers.

Telecommunications Policy, [S.l.], v. 13, n. 3, p. 233-242, 1989. Disponível em: $<$ https://pantherfile.uwm.edu/braman/www/ bramanpdfs/003_defining.pdf $>$. Acesso em: 25 jul. 2018.

BRAMAN, S. Defining Information Policy. Journal of Information Policy, [S.l.], v.1, p. 1-5, 2011. Disponível em:

<https://www.researchgate.net/publication /281600906_Defining_information_policy>. Acesso em: 27 jul. 2018.

BRAMAN, S. A economia representacional e o regime global da política de informação. In: MACIEL, Maria Lúcia; ALBAGLI, Sarita. (Org.) Informação, conhecimento e poder: mudança tecnológica e inovação social. Rio de Janeiro: Garamond, 2011.
BRAMAN, S. The Change of the State: Information, and Policy and Power. Cambridge: MIT press, 2006.

BRAMAN, S. Horizons of the State: Information Policy and Power. Journal of Communication, [S.l.], v. 45, n. 4, p. 4-24, 1995.

BRAMAN, S. The emergent global information policy regime. Houndsmills, UK: Palgrave Macmillan, 2004.

BYGSTAD, B.; HANSETH, O. Governing eHealth Infrastructures: Dealing with Tensions. In: INTERNATIONAL CONFERENCE ON INFORMATION SYSTEMS, 37., Proceedings..., Dublin, 2016.

CONSTANTINIDES, P.; HENFRIDSSON, O.; PARKER, G. G. Introduction Platforms and Infrastructures in the Digital Age.

Information Systems Research, [S.l.], v. 29, n. 2, 2018.

EATON, K. Policy Regime Juxtaposition in Latin America. Colombia Internacional, Bogotá, n. 90, p. 37-65, 2017.

EDWARDS, P. N. et al. Understanding Infrastructure: dynamics, tensions, and Design. Michigan: NSF Grant, 2007.

GONZÁLEZ DE GÓMEZ, M. N. A vinculação dos conhecimentos: entre a razão mediada e a razão leve. Liinc em Revista, Rio de Janeiro, v. 1, n. 1, p. 16-37, 2005. Disponível em: <http://revista.ibict.br/liinc/>. Acesso em: 10 set. 2018 .

GONZÁLEZ DE GÓMEZ, M. N. Da política de informação ao papel da informação na 
política. Revista Internacional de Estudos Políticos. NUSEG/UERJ, Rio de Janeiro, v. 1, n. 1, p. 67-93, 1999.

HABERMAS, J. Between Facts and Norms: Contributions to a discourse theory of law and democracy. Cambridge: MIT Press, 1996.

JOACHIM, A. E.; MAY, P. J. Beyond Subsystems: Policy Regimes and Governance. Policy Studies Journal, [S.l.], v. 38, n. 2, p. 303-327, 2010.

KEOHANE, R. O.; KRASNER. S. Subversive Realist. Presented at the Annual Meeting of the American Political Science Association. Washington, DC, 2010.

KRASNER, S. D. Global Communications and National Power: Life on the Pareto Frontier. World Politics, Cambridge, v. 43, n. 3, p. 336-366, 1991. Disponível em: <http://www.jstor.org/stable/2010398>. Acesso em: 12 ago. 2018.

KRASNER, S. D. Causas estruturais e consequências dos regimes internacionais: regimes como variáveis intervenientes. Revista de Sociologia e Política, Curitiba, v. 20, n. 42, p. 93-110, 2012.

KRASNER, S. D. Structural causes and regime consequences: regimes as intervening variables, International Organization, Massachusetts, v. 36, n. 2, 1982.

MAY, P. J.; JOACHIM, A. E. Policy regime perspectives: policies and governing. In: APPAM Fall Research Conference, Baltimore, 2012. Disponível em: <http://appam.confex.com/appam/2012/w ebprogram/ExtendedAbstract/Paper1585/ MayJochimPReg ime_APPAM2012.pdf>. Acesso em: 6 jun. 2018.

MAY, P. J.; JOACHIM, A. E. Policy regime perspectives: policies, politics, and governing. Policy Studies Journal, [S.l.], v.
41, n. 3, p. 426-452, 2013. Disponível em: <https://onlinelibrary.wiley.com/doi/abs/1 0.1111/psj.12024 >. Acesso em: 7 jun. 2018.

RENEDO, A.; KOMPOROZOS-ATHANASIOU, A.; MARSTON, C. Experience as evidence: the dialogic construction of health professional knowledge through patient involvement. Sociology, [S.l.], v. 52, n. 4, p. 778- 795, 2017.

RODON, J.; HANSETH, O. Stabilization and Destabilization Processes at Work in Digital Infrastructures: the co-functioning of architecture and governance. Working paper. University of Oslo. 2015. Disponível em:<http://heim.ifi.uio.no/ oleha/Publicati ons/CatalanePrescriptionDI030315.pdf>. Acesso em: 10 jun. 2018.

SANTOS, A. C. G.; GONZÁLEZ DE GÓMEZ, M. N. Ações de informação, colaboração e concertação institucional no contexto da extensão rural. Liinc em revista, Rio de Janeiro, v. 10, n. 2, 2014. Disponível em: <http://www.brapci.inf.br/v/a/22838>. Acesso em: 22 jun. 2018.

SANTOS, T. O. Implantação de sistemas informatizados na saúde: uma revisão sistemática. Reciis - Revista Eletrônica de Comunicação Informação e Inovação em Saúde, Rio de Janeiro, v. 11, n. 3, p. 1-11, 2017. Disponível em: $<$ www.reciis.icict.fiocruz.br e-ISSN 19816278.>. Acesso em: 9 jun. 2018.

SILVA, A. B. et al. Registro eletrônico de saúde em hospital de alta complexidade: um relato sobre o processo de implementação na perspectiva da telessaúde. Ciência \& Saúde Coletiva, Rio de Janeiro, 2017. Disponível em:<https://bit.ly/2DHRvFg>. Acesso em: 22 ago. 2018.

SILVA, A. B.; MOREL, C. M.; SOZZI, M. I. Proposta conceitual de telessaúde no modelo da pesquisa translacional. Revista de Saúde Pública, Rio de Janeiro, v. 48, n. 2, p. 347356, 2014. 


\title{
NOTAS
}

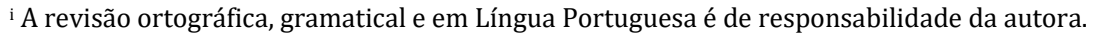

ii Para Sandra Braman $(2004,2006)$, as tecnologias se diferenciariam conforme quatro dimensões: o grau em que são "sociais" em sua produção e uso; a complexidade do processo capazes de realizar; seu grau de autonomia; a escala de sua manifestação. De acordo com essas variações, a história das tecnologias apresentaria três grandes "famílias" de técnicas a serviço do fazer humano (making): as ferramentas, que podem ser usadas por um único homem, como o martelo; as tecnologias propriamente ditas, que são dispositivos sociais em sua produção e uso, mas cada uma delas com uma linha sequencial e diferenciada de processamento e transformação dos materiais, e as meta-tecnologias.
\end{abstract}

iii Bendik Bygstad (bendikby@ifi.uio.no), e Ole Hanseth (oleha@ifi.uio.no) Department of Informatics, University of Oslo, Oslo, Norway, desenvolvem pesquisa sobre infraestruturas de informação e sistemas de informação que atendem a macro-sistemas de atendimento à saúde. Destacamos estas pesquisas por um lado, pelo conhecimento prévio dos autores, e por outro, porque trazem a experiência com sistemas estatais de saúde.

iv "How should this challenge be governed in e-health? A combination of two approaches have been chosen in most countries: The establishment of an IT governance regime, in order to plan, prioritize and coordinate the various activities (Weill and Ross, 2004; ISACA 2012).The establishment of an IT architectural regime, consisting of enterprise architecture (EA), various standards and an implementation process (Ross et al., 2006; Open Group, 2011).” Bygstad; Hanseth (2016), p. 2. 